\title{
Evidence of fractional-Brownian-motion-type asperity model for earthquake generation in candidate pre-seismic electromagnetic emissions
}

\author{
K. Eftaxias ${ }^{1}$, Y. Contoyiannis ${ }^{2}$, G. Balasis ${ }^{3}$, K. Karamanos ${ }^{1}$, J. Kopanas ${ }^{1}$, G. Antonopoulos ${ }^{1}$, G. Koulouras ${ }^{4}$, and \\ C. Nomicos $^{4}$ \\ ${ }^{1}$ Section of Solid State Physics, Department of Physics, University of Athens, Panepistimiopolis, Zografos, \\ 15784, Athens, Greece \\ ${ }^{2}$ Section of Nuclear and Elementary Particle Physics, Department of Physics, University of Athens, Panepistimiopolis, \\ Zografos, 15784, Athens, Greece \\ ${ }^{3}$ Institute for Space Applications and Remote Sensing, National Observatory of Athens, Metaxa and Vas. Pavlou, Palea \\ Penteli, 15236, Athens, Greece \\ ${ }^{4}$ Department of Electronics, Technological Educational Institute of Athens, Ag. Spyridonos, Egaleo, 12210, Athens, Greece
}

Received: 4 April 2008 - Accepted: 5 June 2008 - Published: 11 July 2008

\begin{abstract}
Many aspects of earthquake generation still escape our full understanding. Observations of electromagnetic emissions preceding significant earthquakes provide one of the few cases of premonitory events that are possibly related to a subsequent earthquake. Understanding the factors that control electromagnetic precursors generation seems to be important for determining how significant earthquakes nucleate. Here we report the results of a comprehensive study of the appearance of individual patterns in candidate electromagnetic precursors possibly indicating the breaking of backbone of large and strong asperities that sustain the activated fault. The search of precursory patterns is mainly based on well documented scaling properties of fault surface topology. More precisely, we argue that the candidate electromagnetic precursors might be originated during the slipping of two rough and rigid fractional-Brownian-motion-type profiles one over the other, with a roughness which is consistent with field and laboratory studies. The results also imply that the activation of a single earthquake (fault) is a reduced selfaffine image of the whole regional seismicity and a magnified self-affine image of the laboratory seismicity.
\end{abstract}

\section{Introduction}

Earthquakes (EQs) are large-scale fracture phenomena in the heterogeneous crust of the Earth. Despite the large amount of experimental data and the considerable effort that has been

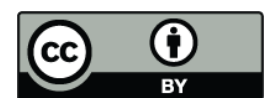

Correspondence to: K. Eftaxias (ceftax@phys.uoa.gr) undertaken by the material scientists, funtamental questions about fracture processes remain standing. Especially, many aspects of EQ generation still escape our full understanding.

Crack propagation is the basic mechanism of material failure. The motion of a crack has been shown to be governed by a dynamical instability causing oscillations in its velocity and structure on the fracture surface (Bahat et al., 2005; Contoyiannis et al., 2005 and references therein). Experimental evidence indicates that the instability mechanism is that of local branching: a multi-crack state is formed by repetitive, frustrated micro-fracturing events. The rupture of inter-atomic (ionic) bonds also leads to intense charge separation. On the faces of a newly created micro-crack the electric charges constitute an electric dipole or a more complicated system. Due to the strong wall vibration in the stage of the micro-branching instability, crack behaves as an efficient electromagnetic (EM) emitter. Thus, when a material is strained, EM emissions in a wide frequency spectrum ranging from $\mathrm{kHz}$ to $\mathrm{MHz}$ are produced by opening cracks, which can be considered as the so-called precursors of general fracture; these precursors are detectable both at a laboratory (Bahat et al., 2005; Rabinovitch et al., 2007; Hadjicontis et al., 2007) and a geological scale (Eftaxias et al., 2007a and references therein).

Our main observational tool is the monitoring of the fractures which occur in the focal area before the final breakup by recording their kHz-MHz electromagnetic emissions. Clear kHz-to-MHz EM anomalies have been detected over periods ranging from a few days to a few hours prior to recent destructive EQs in Greece, with the MHz radiation appearing earlier than the kHz. Recent results indicate that these precursory EM time-series contain information characteristic of

Published by Copernicus Publications on behalf of the European Geosciences Union. 


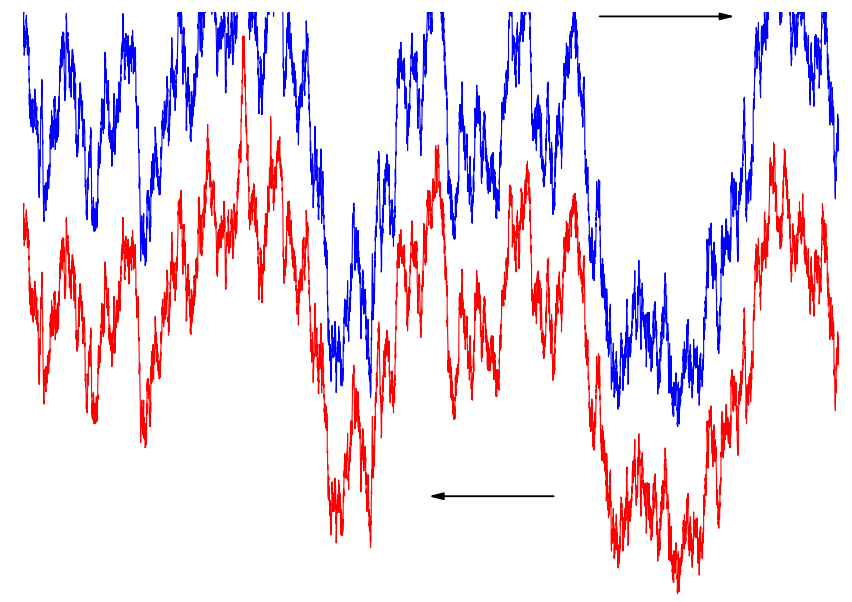

Fig. 1. Fault planes realized by two Brownian profiles.

an ensuing seismic event (Kapiris et al., 2004a, 2004b; Contoyiannis et al., 2005; Karamanos et al., 2005, 2006; Eftaxias et al., 2007b; Papadimitriou et al., 2008).

We underline that the $\mathrm{MHz}$ radiation systematically appears earlier than the $\mathrm{kHz}$ activity in both laboratory and geophysical scale (Eftaxias et al., 2002). Motivated by this experimental evidence, recently we have attempted to identify these two distinct epochs of precursory EM activity with the equivalent last stages in the EQ preparation process. In this direction, our model of the focal area consists of: (i) a backbone of strong and large asperities distributed along the activated fault; and (ii) a strongly heterogeneous medium, including weaker areas and smaller asperities, that surrounds the family of main asperities. Based on aspects rooted in criticality, spectral fractal analyses by means of wavelets, complexity, non-extensive statistics, and mesomechanics we proposed the following two epochs / stages model (Kapiris et al., 2004a; Contoyiannis et al., 2005; Eftaxias et al., 2007b; Papadimitriou et al., 2008): The first epoch, which includes the initially emerged $\mathrm{MHz}$ part, originates during cracking in the highly heterogeneous component of the focal area. The results shown that in the candidate $\mathrm{MHz}$ EM precursor has been projected a fracture process which undergoes as a generalized continuous phase transition at equilibrium. The second epoch, which includes the finally emerged strong multipeaked $\mathrm{kHz}$ EM radiation, is due to the fracture of the high strength large asperities that sustain the system. We emphasize that we did not find any signature of a generalized continuous phase transition in the $\mathrm{kHz}$ EM activity (Contoyiannis et al., 2005).

In the present study we attempt to further support the above mentioned two stage model. In particular, we focus on the second crucial stage. We attempt to establish the hypothesis that the sequence of precursory $\mathrm{kHz}$ EM pulses ("electromagnetic earthquakes") is induced by the slipping of two rough and rigid Brownian profiles one over the other (Fig. 1).

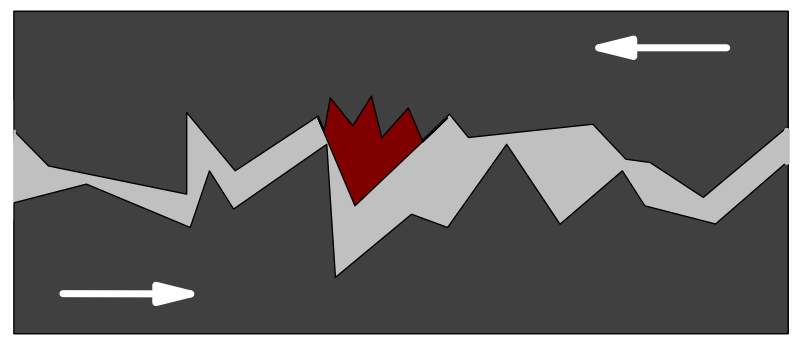

Fig. 2. Fault planes realized by two Brownian profiles put in contact at one point. An electromagnetic pulse ("electromagnetic earthquake") occurs when there is an overlap of the two profiles representing the fault faces.

In this scheme, an individual "electromagnetic earthquake" is emitted when there is an intersection between the two Brownian profiles of the single activated fault (see Fig. 2).

Ample experimental and theoretical evidence support the above mentioned hypothesis: (i) kinematic or dynamic source inversions of EQs suggest that the final slip (or the stress drop) has a heterogeneous spatial distribution over the fault (see among others Gusev, 1992; Bouchon, 1997; Peyrat et al., 2001). (ii) Power spectrum analysis of the fault surface suggests that heterogeneities are observed over a large range of scale lengths (see Power et al., 1987, in particular Fig. 4). (iii) Investigators of the EQ dynamics have already pointed out that the fracture mechanics of the stressed crust of the earth forms self-similar fault patterns, with welldefined fractal dimensionalities (Kagan et al., 1982; Sahimi et al., 1993; Barriere and Turcotte, 1991). (iv) Following the observations of the self-similarity in various length scales in the roughness of the fractured solid surfaces (Chakrabarti, B. and Benguigui, 1997; Chakrabarti, B. and Stinchocombe, 1999) have proposed that the contact area distribution between two fractal surfaces follows a unique power law.

\section{The $\mathrm{kHz}$ precursory EM radiation associated with the Athens earthquake}

On 7 September 1999 the Athens EQ $\left(38.2^{\circ} \mathrm{N}, 23.6^{\circ} \mathrm{E}\right)$ with a magnitude $M=5.9$ occurred. Pre-seismic EM anomalies at $\mathrm{MHz}$ and $\mathrm{kHz}$ frequency bands emerged during the last 7 days prior to seismic shock; the MHz EM candidate precursor appeared earlier than the $\mathrm{kHz}$ (Contoyiannis et al., 2005). In particular, clear $3 \mathrm{kHz}$ and $10 \mathrm{kHz}$ had been simultaneously recorded during the last few days prior to this EQ (Eftaxias et al., 2001). They ceased a few hours before the Athens EQ occurrence.

In Fig. 3 we present the EM time series at $10 \mathrm{kHz}$ (EastWest) from 4 July 1999 up to 12 September 1999: the emerged EM precursor is characterized by an accelerated emission rate, while, it is embedded in a long duration 


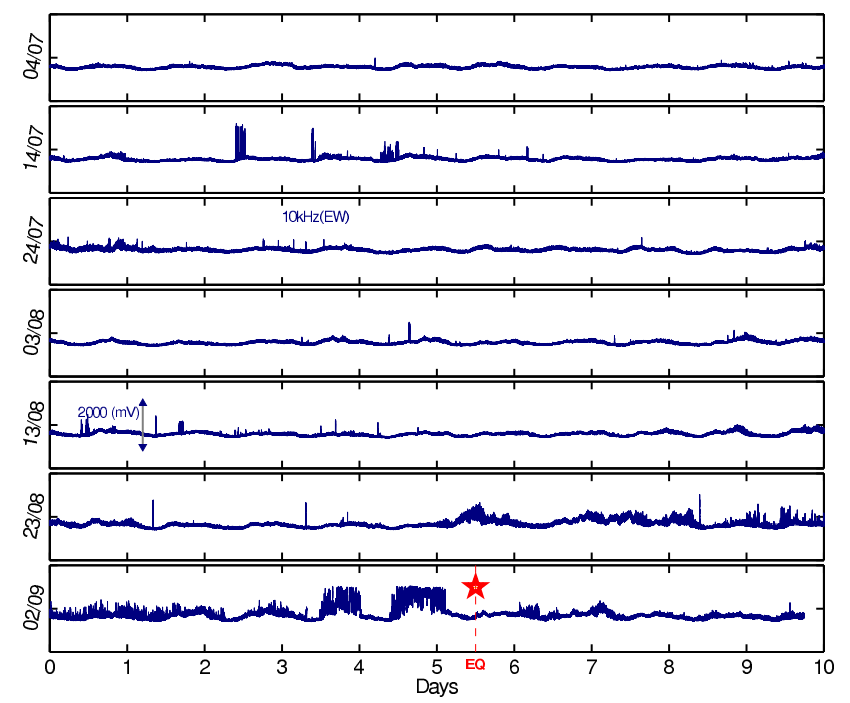

Fig. 3. Time series of the $10 \mathrm{kHz}$ (East-West) magnetic field strength between 4 July 1999 and 11 September 1999 in arbitrary units. The star indicates the time of the Athens earthquake occurrence. The EM precursor (see Fig. 4) is characterized by an accelerated emission rate, while, this radiation is embedded in a long duration quiescence period. We underline the presence of two strong impulsive electromagnetic burst at the tail of the candidate precursor.

quiescence period. In Fig. 4, we show the same EM time series from 28 August 1999 up to 8 September 1999.

We mainly focus on the $\mathrm{kHz}$ precursory EM emissions associated with the Athens EQ for the following reasons: (i) It has a long duration, thus, it provides sufficient data for a valuable statistical analysis; the data have been recorded with a sampling rate of 1 sample/sec while the duration of the candidate $\mathrm{kHz}$ EM precursor is more than six days. (ii) $\mathrm{A}$ multidisciplinary analysis in terms of fault modeling, laboratory experiments, scaling similarities of multiple fracturing of solid materials, fractal electrodynamics, criticality, complexity, and mesomechanics seems to validate the association of the detected pre-seismic EM emission with the fracturing process in the focal area of the impending EQ (Eftaxias et al., $2007 \mathrm{~b}$ and references therein).

\section{Signatures of two Brownian profiles modeling the fault surfaces in pre-seismic $\mathrm{kHz}$ EM emissions}

Maslov et al. (1994) have formally established the relationship between spatial fractal behavior and long-range temporal correlations for a broad range of critical phenomena. They showed that both the temporal and spatial activity can be described as different cuts in the same underlying fractal. A self-organized critical process, as the source of the temporal power-laws, further suggests that similar power-laws

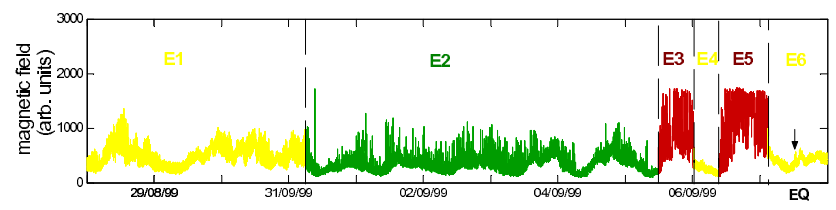

Fig. 4. The green and yellow time series refers to the candidate EM precursor following the antipersistent fractional Brownian motion model and persistent fractional Brownian motion model. The electromagnetic background (noise) follows the fractional Gaussian noise model. Moreover, the yellow epoch is characterized by a high complexity, while, the green epoch shows a decrease of complexity in comparison to that of the yellow epoch, and the red epoch shows a significant decrease of complexity in comparison to those of the yellow and green epochs.

also exist for parameters in the spatial domain (Hansen and Schmittbuhl, 2003). Laboratory experiments support the consideration that both the temporal and spatial activity can be described as different cuts in the same underlying fractal. Characteristically, Ponomarev et al. (1997) have studied the temporal evolution of Hurst exponent for the series of distances $H_{r}$ and time intervals $H_{t}$ between consecutive acoustic emission events in rocks. Their analysis indicates that the changes of $H_{r}$ and $H_{t}$ with time occur in phase, while the relationship $H_{r} \approx H_{t}$ is valid.

In this section, in the frame of the above mentioned scheme, we investigate whether the KHz EM precursor behaves as a temporal fractal following the fractional Brownian motion model.

\subsection{Application to the data}

If a pre-seismic EM time series is a temporal fractal, then, a power-law of the form $S(f) \propto f^{-\beta}$ is obeyed, with $S(f)$ the power spectral density (PSD) and $f$ the frequency. In a $\log S(f)-\log f$ representation the power spectrum is a line with slope $\beta$. The spectral scaling exponent $\beta$ is a measure of the strength of time correlations.

The "wavelet spectrum" is used in order to provide an unbiased and consistent estimation of the true power spectrum of the time-series. The continuous wavelet tranform based on the Morlet wavelet makes the calculation. Herein, we use a 1024 moving window and an overlap of one sample. For each window the local parameter $\beta$ was derived. In Fig. 5 the time series and the values of the spectral parameter $\beta$ are plotted.

Two classes of signal have been widely used to model stochastic fractal time series: fractional Gaussian noise (fGn) and fractional Brownian motion (fBm) (Heneghan and McDarby, 2000). For the case of the fGn model the scaling exponent $\beta$ lies between -1 and 1 , while the regime of $\mathrm{fBm}$ is indicated by $\beta$ values from 1 to 3 . 


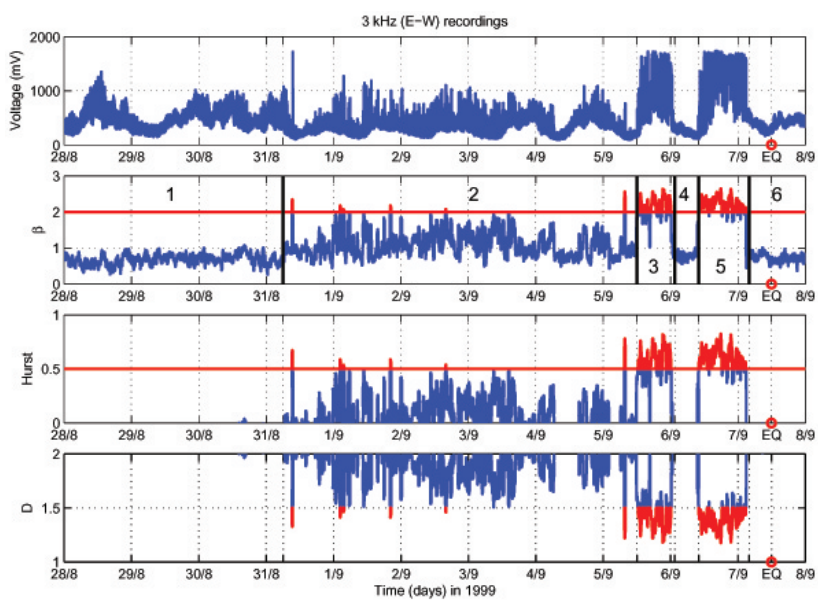

Fig. 5. From top to bottom: Electromagnetic time series (upper panel); evolution of the $\beta$ exponent, Hurst exponent and fractal dimension, $D$, with time (lower panels).

Figure 5 shows that far from the EQ occurrence (epoch 1 in Fig. 5) $\beta$ takes values between 0 and 1 indicating that the EM background (noise) follows the fGn model. The epochs 4, 6 in Fig. 5 also follow the fGn model representing the EM background, too. The candidate EM precursor clearly emerged from the EM background (epochs 2, 3, 5 in Fig. 5) following the $\mathrm{fBm}$ model. Indeed, the $\beta$ exponent in these windows takes values between 1 and 3 .

Consequently, the profile of the candidate $\mathrm{kHz}$ EM precursor is qualitatively analogous to $\mathrm{fBm}$ model. This finding quantitatively suggests that the sequence of precursory EM pulses could be originated during the slipping of two rough and rigid Brownian profiles one over the other.

\section{On a relationship between "roughness" of fracture surfaces and "roughness" of pre-seismic EM time se- ries}

One of the most important challenges is to make not only a quantitative link but also a quantitative comparison between pre-seismic EM time series and fracture surfaces. We attempt such a comparison by means of "roughness" of preseismic time series profile on one hand and "roughness" of topography of fracture surfaces on the other hand. Importantly, various fractographic investigations indicate a fairly robust universal behavior for fracture surfaces. Thus, an interesting question is whether the roughness exponent of the preseismic time series is in agreement with the universal value of roughness of fracture surfaces.

Fracture surfaces were found to be self-affine over a wide range of length scales. Therefore, the height-height correlation function $\Delta h(r)=<[h(r+\Delta r)-h(r)]_{r}^{\frac{1}{2}}>$ computed along a given direction is found to scale as $\Delta h \sim(\Delta r)^{H}$, where $H$ refers to the Hurst exponent. $H$ specifies the strength of the irregularity ("roughness") of the fBm surface topography: the fractal dimension is calculated from the relation $D=(2-H)$ (Heneghan and McDarby, 2000). More precisely, the "roughness" exponents $H$ expresses the tendency for $d h=[d h(x) / d x] d x$ to change sign. When $\frac{1}{2}<H<1$, the sign tends not to change. The value $H=1$ is an upper bound reached when the "roughness" of the fault is minimum, in other words, a differentiable surface topography corresponds to $H=1$. For $0<H<\frac{1}{2}$ there is a tendency for the sign to change (anticorrelation). The value $H=0$ is a lower bound; as $H$ tends towards 0 trends are more rapidly reversed giving a very irregular look. Surfaces with $H>\frac{1}{2}$ are said to be persistent, and those with $<\frac{1}{2}$ are anti-persistent. When $H=\frac{1}{2}$, the sign of $d h$ changes randomly, and the corresponding surface possesses no spatial correlations.

$H$-exponent also specifies the strength of the irregularity ("roughness") of fBm profiles of time series.

4.1 On the "roughness" of the candidate precursory EM time series

First we focus on the candidate EM precursor under study. The $\beta$ exponent is related to the Hurst exponent, $H$, by the formula $\beta=2 H+1$, with $0<H<1(1<\beta<3)$ for the fBm model (Heneghan and McDarby, 2000). We distinguish two different epochs in the candidate precursory emission. The first epoch (epoch 2 in Fig. 5) is characterized by an antipersistency, $0<H<0.5(1<\beta<2)$, reflecting that if the fluctuations increase in a period, it is likely to decrease in the interval immediately following and vice versa. This means that the "roughness" of the profile is high. This epoch might to refer to the fracture of weaker and smaller asperities. The anti-persistent character may reflect the fact that weak and small asperities with a low threshold for breaking encounter stronger asperities in the direction of crack propagation. When this happens, cracking growth / EM emission stops, and then starts again at a later time in the weaker of the remaining undamaged sections, and so on. Finally, only the fraction of high strength and large asperities remain along the fault sustaining the system. The second and last epoch of the candidate precursor (epochs 3 and 5 in Fig. 5) is characterized by an abrupt onset of strong burst-like EM emission. We argue that this emission witnesses the fracture of the population of stronger and larger asperities (Kapiris et al., 2004a; Contoyiannis et al., 2005; Papadimitriou et al., 2008). It is characterized by persistency, $0.5<H<1(2<\beta<3)$, which means that if the amplitude of fluctuations increases in a time interval it is likely to continue increasing in the interval immediately following. This means that the "roughness" of the profile is low. This feature is in harmony with a positive feedback of broken asperities, which increases the stress and strain on the unbroken elements leading to the global rupture of the system. In other words, the system tends toward irreversibility. The aforementioned hypothesis is supported 
by the fact that the launch of persistency is coupled with (i) a power-law type acceleration of the EM energy release (Kapiris et al., 2004a; Contoyiannis et al., 2005); (ii) a transition to a significantly more ordered state (red epoch), which has been verified by various measures of complexity, e.g., Kolmogorov-Sinai Entropy, Block Entropy, T-Entropy, Approximate Entropy, Correlation Dimension, Tsallis Entropy further indicating that any small instability can provoke large scale reactions (Karamanos et al., 2005, 2006; Kalimeri et al., 2008; Papadimitriou et al., 2008).

We pay attention to the following experimental evidence. Recently, statistical physicists have shown the existence of a power-law acceleration of acoustic emissions announcing the global rupture, similar to the critical behavior of the out of equilibrium phase transition, offering a way to predict material rupture (Sornette and Helmstetter, 2002). Notice, this critical phenomenon has been also observed prior to a rock failure in terms of EM energy release (Rabinovitch et al., 2001). The rate of both the seismic and EM energy release were exhibited a significant power-law type increase during the last days before the EQ occurence (Kapiris et al., 2004a; Eftaxias et al., 2007b, Fig. 12). This evidence supports the hypothesis that the detected EM precursor is a subproduct of the Athens fault system nucleation. Importantly, the fault modeling of the Athens EQ, based on information obtained by radar interferometry (ERS-2 satellite) (Kontoes et al., 2000), predicts two faults: the main fault segment is responsible for $80 \%$ of the total energy released, with the secondary fault segment for the remaining $20 \%$. On the other hand, the first strong EM burst at the tail of the associated $\mathrm{kHz}$ preseismic EM emission (Figs. 3, 4) contains approximately $20 \%$ of the total EM energy received during the emergence of the two bursts, and the second the remaining $80 \%$ (Eftaxias et al., 2001). This surprising correlation in the energy domain between the two strong pre-seismic $\mathrm{kHz}$ EM signals and two faults activated in the case of the Athens EQ, strongly supports the hypothesis that the detected two strong EM bursts might be rooted in the fracture of two families of main asperities associated with the two activated faults in the case of the Athens EQ (Eftaxias et al., 2001).

We emphasize that a few days prior to the Athens event, the seismicity was centered at a distance of about one source dimension $(<30 \mathrm{~km})$ from the epicentre (Kapiris et al., 2005b and references therein). Laboratory studies and theoretical arguments suggest that the damage localization and sensitivity of energy release characterize the fracture surface formation, and thus provide two cross-checking precursors for the prediction of rupture (Dodze at al., 1996; Reasenberg, 1999; Li et al., 2002).

The above mentioned experimental findings suggest that the possible geometrical scaling inherent in the structure of the activated two faults has been more clearly projected in the second strong EM burst. We focus on this burst. Figure 6 shows the $\log S(f)-\log f$ representation of the power spectrum for the whole second strong EM burst. The associated $\beta$ value is 2.51 , which leads to the global roughness $H=0.75$. Figure 5 shows that the local Hurst exponents are distributed around this value. This finding predicts that the activated main fault in the case of the Athens EQ might be consisted by two rough and rigid Brownian profiles which are characterized by a roughness distributed around the value $H=0.75$. In the next section we examine whether this prediction is supported by laboratory and field experimental data and theoretical argument.

4.2 On the "roughness" of the topography of fracture surfaces

Herein, we concentrate on the question whether a quantitative relationship exists between the Hurst-exponents that control the "roughness": (i) of topography of fracture surfaces, on one hand, and (ii) profiles of pre-seismic EM time series, on the other hand.

The study of the morphology of fracture surfaces is nowadays a very active field of research. From the early work of Mandelbrot (1982), much effort has been put into the statistical characterization of the resulting fractal surfaces in fracture processes. Importantly, it has been pointed out that natural rock surfaces can be represented by fractional Brownian surfaces over a wide range (Huang and Turcotte, 1988). It should be stressed that experimental results in very different types of materials (from ductile aluminum alloys, silica glasses to brittle materials like rock) seem to support the idea of a universal roughness exponent. Indeed, the Hurst exponent $H \sim 0.75-0.8$ has been interpreted as a universal indicator of surface fracture, weakly dependent on the nature of the material and on the failure mode (Lopez and Schmittbuhl, 1998; Hansen and Schmittbuhl, 2003; Ponson et al., 2006).

An important technique to determine the critical exponents of a growing surface is to study the Fourier transform of the interface height. In this representation, the properties of the surface can be investigated by calculating the power spectrum $S(k, t)$, where $k$ is the spatial wave number. Lopez and Schmittbuhl (1998) and Mourot et al. (2006) have studied the scaling behavior of the local fluctuations of a brittle fracture in a granite block and mortar crack surfaces, correspondingly. They found that the power spectrum $S(k)$ decays with a power law $k^{-2.58}$, for various times $t$, which is consistent with a local roughness exponent 0.79 . We recall that the second EM burst associated with the activation of the main fault is characterized by the power spectrum $S(f) \propto f^{-\beta}$ with $\beta$ equal to 2.51 , which leads to a roughness exponent 0.75 .

The surface roughness of a recently exhumed strike-slip fault plane has been measured by three independent 3D portable laser scanners (Renard et al., 2006). This fault plane refers to the Vuache fault, near Annecy in the French Alps. Statistical scaling analyses show that the striated fault surface exhibits self-affine scaling invariance with a small directional morphological anisotropy that can be described by 


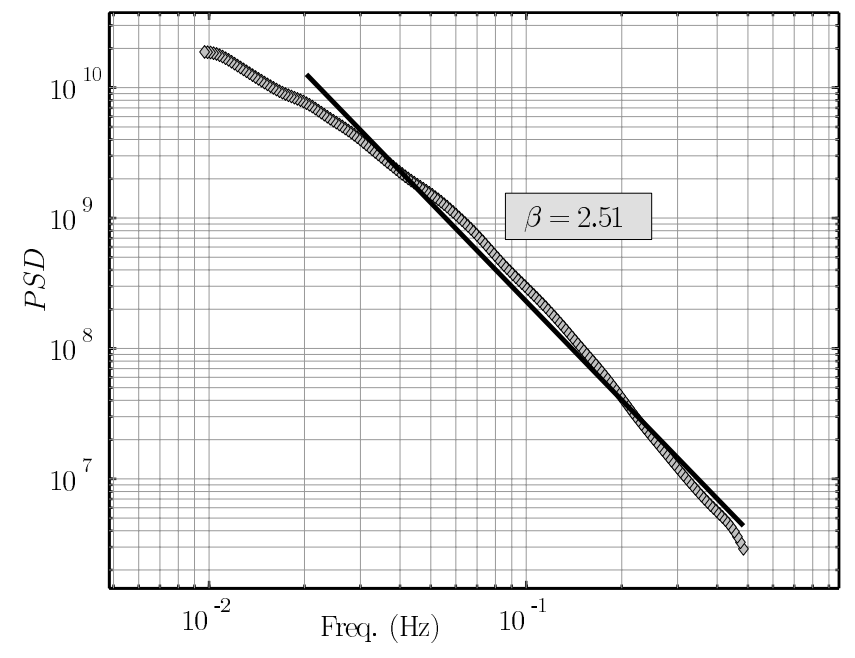

Fig. 6. A $\log -\log$ representation of the relation $S(f)=f^{-\beta}$ for the second strong EM burst emerged at the tail of the candidate precursor (see Figs. 3, 4). We observe that this equation fits very well the data.

two scaling roughness exponents, $H_{1}=0.7$ in the direction of slip and $H_{2}=0.8$ perpendicular to the direction of slip.

Finally, Zapperi et al. (2005) have analyzed the scaling of the crack roughness in the two-dimensional random fuse model by numerical simulations. A fit of the power law decay of the spectrum leads to roughness 0.8 for diamond lattices.

In summary, laboratory and field experimental data, as well as numerical studies suggest the self-affinity in the roughness of the fractured solid surfaces. The performed analysis demonstrate that the roughness of the profile of preseismic time series under study practically coincides with the universal roughness of fracture surfaces.

\subsection{Analysis in terms of fractal dimension}

As it is said, the fractal dimension $D$ also specifies the strength of the irregularity of the $\mathrm{fBm}$ surface topography: the fractal dimension is calculated from the relation $D=(2-H)$ (Heneghan and McDarby, 2000). Using acoustic emission data, Feng and Seto (1999) showed that a larger fractal dimension corresponds to a more stable state of the system, while just prior to failure the fractal dimension decreases quickly to lower values. Notice, this result is consistent with the conclusion drawn from the spatial distribution of rock bursts and EQs, that the lower fractal dimension occurs near the time of a strong EQ (Sammods et al., 1992). Figure 5 shows the temporal evolution of fractal dimension $D$ in the candidate EM precursor. The observed significant reduction of $D$ in the tail of the preseismic EM emission, i.e., within the two strong EM bursts, further indicates the underlying unstable state. In the second burst we have $D=1.25$.
Notice, seismological measurements as well as theoretical studies (Sahimi et al., 1993) suggest that a surface trace of a single fault might be characterized by $D \sim 1.2$. It further implies that both the temporal and spatial activities can be described as different cuts in the same underlying fractal. On laboratory scale, Lei et al. (2000) and Lei and Satoh (2007) have studied in terms of acoustic emission how an individual asperity fractures, how coupled asperities fracture, and also the role of asperities in fault nucleation and as potential precursors prior to dynamic rupture. The fractal dimension decreases to $\sim 1.0-1.4$ during asperity fracture. The estimated $D$ values close to 1 meets the notion generally accepted that the terminal phase of fracture process is accompanied by a significant increase in localization and directionality. Importantly, Hirata et al. (1987) found that the spatial distribution of the hypocenters of the opening cracks shows fractal structure, however, the fractal dimension significantly decreases with the evolution of the micro-fracturing process. Recently, Kuksenko et al. (2007) based on rock fracture experiments, showed that the Hurst analysis of the space-time distributions of acoustic signals emitted from the compressed samples of granite indicates a clear pronounced trend to planar organization of final damage structure.

The observed gradual decrease of $D$-values coupled with the fact that the frequency of large EM events are relatively enhanced to small ones, is consistent with the following physical picture. As the surfaces move together, contacts become more closely spaced and formation of large contacts occurs at increasing rates. Hence, as the total area of contact increases there is a progressive decrease of roughness of the fault profile and an increase in the numbers of larger contacts / EM events relative to small contacts / EM events.

\section{The activation of a single EQ (fault) as a reduced self- affine image of the whole regional seismicity}

Huang and Turcotte (1988) have pointed out that the statistics of seismicity could be merely a macroscopic reflection of the physical processes in EQ source. In this section, we attempt to check this suggestion.

\subsection{Arguments in terms of a self-affine model of regional seismicity}

De Rubeis et al. (1996) and Hallgass et al. (1997) have introduced a regional fault dynamics by means of the slipping of two rough and rigid Brownian profiles one over the other. In this scheme, an individual EQ occurs when there is an intersection between the two profiles. This model exhibits a good interpretation of the seismicity generated in a large geographic area usually identified as "seismic region", covering many geological faults, on a global sense. Hallgass et al. (1997) have drawn attention to the fact that "what is lacking is the description of what happened locally, i.e., as 
a consequence of a single event, from both the temporal and the spatial point of view". Herein, we focus on the activation of a single fault.

On the other hand, Huang and Turcotte (1988) have pointed out that the statistics of seismicity could be merely a macroscopic reflection of the physical processes in EQ source. Generally, the self-similar nature of faulting and fracture is widely documented from the analysis of data from both field observations and laboratory experiments (Sornette, 2004).

Stimulated by these considerations, we study whether the activation of a single EQ (fault) is a reduced self-affine image of the whole regional seismicity. Moreover, we examine whether the activation of a single EQ (fault) is a magnified self-affine image of the laboratory seismicity.

The self asperity model introduced in Hallgass et al. (1997) predicts that the distribution of areas of the asperities broken $A$ follows a power law

$P(A) \sim A^{-\delta}$

with an exponent $\delta$ which could be related to the Hurst exponent $0<H<R 1$ that controls the roughness of the fault. The former relation is obtained by supposing that the area of the broken asperities scales with its linear extension $l$ as $A_{\text {asp }} \sim l^{(1+H)}$. Based on the above mentiond it is reasonable to assume that the broken asperities scales with its linear $l$ as $A_{\text {asp }} \sim l^{1.75}$. Numerical studies indicate that the number of bonds that break scales during the whole process of fracture as $l^{1.7}$ with the system size $l$ (De Arcangelis et al., 1989). The observed similarity verifies the equality between the roughness of the profiles of the fault and the roughness of the associated EM time series.

The self affine asperity model (Hallgass et al., 1997) also reproduces the Gutenberg-Richter (G-R) law. It predicts that a seismic event releases energy in the interval $[E, E+d E]$ with a probability $P(E) d E, P(E) \sim E^{-B}$, where $B=\alpha+1$ and $\alpha=1-H / 2$ with $\alpha \in\left[\frac{1}{2}, 1\right]$. We recall that the distribution of energies released at any EQ is well described by the power-law, $P(E) \sim E^{-B}$, where $B \sim 1.4-1.6$ (Gutenberg and Richter, 1954). In our case, we have $H=0.75$, which leads to $\alpha \sim 0.67$, and thus, the fracture of asperities release EM energies following the distribution $P(E) \sim E^{-B}$, where $B \sim 1.67$.

The above mentioned findings further verify the hypothesis that: (i) the activation of a single EQ (fault) is a reduced self-affine image of the whole regional seismicity; and (ii) the scaling hidden in the detected $\mathrm{kHz}$ EM precursor could be rooted in a fault topography following power law scaling.

\subsection{Arguments in terms of Gutenberg-Richter law}

The classic power-law found in EQs catalogues is the G$\mathrm{R}$ magnitude-frequency relationship: the cumulative number of EQs with magnitude greater than $M$ is given by

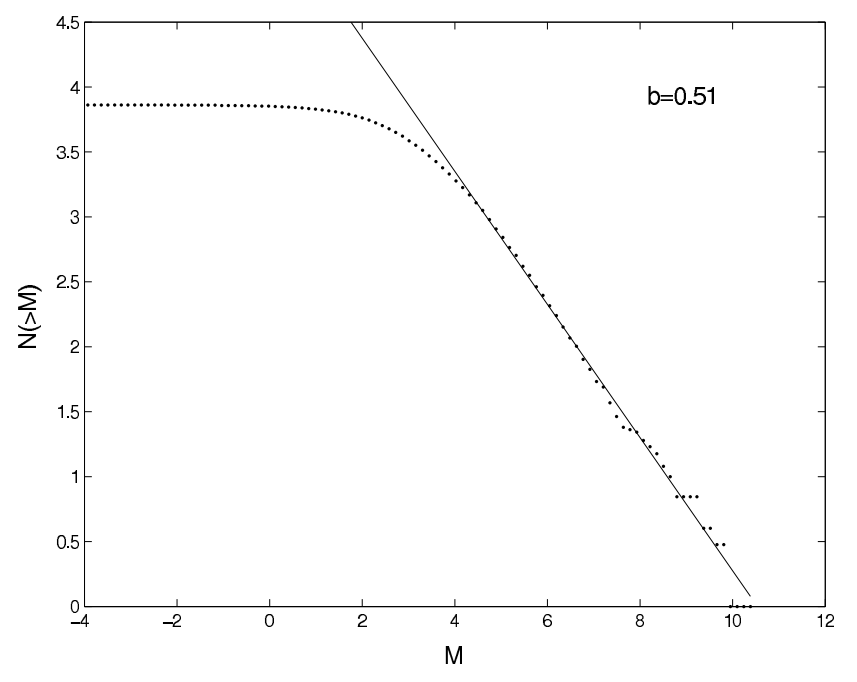

Fig. 7. Number of "electromagnetic earthquakes" (see text) with magnitude $M$ higher that given by the corresponding abscissa. The continuous line is the least squares fit of the power law $N(>M)=M^{-b}$, where $b=0.51$.

$\log N(>M)=\alpha-b M$, where the constant $\alpha$ characterizes the overall rate of activity in a region. There are increasing reports on premonitory decrease of b-value before EQs: foreshock sequences and main shocks are characterized by a much smaller exponent compared to aftershocks, $b \sim 1$ (Hainzl et al., 2003). Importantly, G-R law also holds for acoustic emission events in rock samples (Scholz, 1968). Acoustic emissions from rock fracturing also show a significant decrease in the level of the observed b-values immediately before the global fracture. Recently, Lei and Satoh (2007), based on acoustic emission events recorded during the catastrophic fracture of typical rock samples under differential compression, suggest that the pre-failure damage evolution is characterized by a dramatic decrease in $b$-value from $\sim 1.5$ to $\sim 0.5$ for hard rocks. Laboratory experiments in terms of acoustic emission performed by Ponomarev et al. (1997) also showed a significant fall of the observed b-values from $\sim 1$ to $\sim 0.6$ just before the global rupture.

Herein, we focus on the activation of a single fault in terms of preseismic EM radiations. The background (noise) level of the EM time series $A\left(t_{i}\right)$ is $A_{\text {noise }}=500 \mathrm{mV}$ Kapiris et al. (2004b). We regard as amplitude $A$ of a "seismogenicEM emission" the difference $A_{\text {fem }}\left(t_{i}\right)=A\left(t_{i}\right)-A_{\text {noise }}$.

We consider that a sequence of $k$ successively emerged "EM-emissions" $A_{\text {fem }}\left(t_{i}\right), \quad i=1, \ldots, k$, namely an EM avalanche, represents the EM energy released, $\varepsilon$, during the damage of an asperity (see Fig. 2). We shall refer to this as an " electromagnetic earthquake". The squared amplitude of seismogenic-EM emissions is proportional to their energy, $\epsilon$. Therefore, the magnitude $M$ of an "electromagnetic earthquake" is given by the relation $M=\log \varepsilon \sim \log \left(\sum\left[A_{f e m}\left(t_{i}\right)\right]^{2}\right)$. 


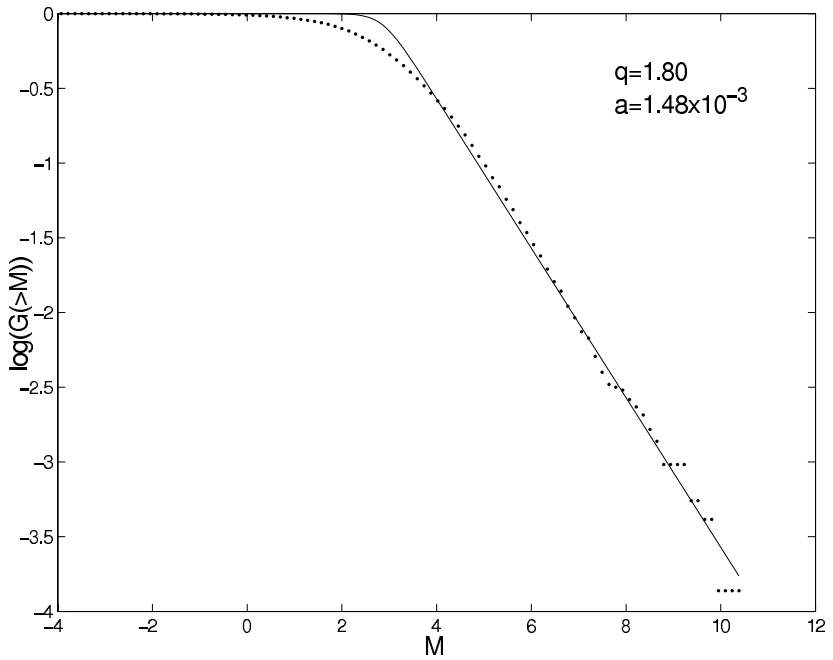

Fig. 8. We use formula (1) to calculate the relative cumulative number of "electromagnetic earthquakes" (see text), $G(>M)$, included in the whole precursory phenomenon, namely, into the green and red epochs depicted in Fig. 4. There is an agreement of formula (1) with the data.

Figure 7 shows the quantity $N(>A)$ vs $M$, where $N(>A)$ is the cumulative number of the detected "electromagnetic earthquakes" with magnitude greater than $M$. The main part of this distribution is given by $\log N(>M)=\alpha-b M$, where $b \sim 0.5$. This finding further implies that a single EQ behaves as a reduced image of natural regional seismicity and a magnified image of laboratory seismicity.

We note that Scholz (1968), based on laboratory experiments, suggested that the changes of $b$-value are inversely proportional to changes in the ambient stress level. The $b$ value of EQs has also been shown to be inversely dependent of shear stress (Wiemer and Schorlemmer, 2007). In particular, asperities are found in many c ase studies to be characterized by low $b$-values (Schorlemmer and Wiemer, 2005; Wiener and Schorlemmer, 2007 and references therein). It might be concluded that the low $b$-value associated with the sequence of precursory "electromagnetic earthquakes" implies that high stresses siege the backbone of asperities.

\subsection{Arguments in terms of non-extensive statistics}

A model for EQ dynamics consisting of two rough profiles interacting via fragments filling the gap, analogous to that introduced in De Rubeis et al. (1996), has been recently introduced by Solotongo-Costa and Posadas (2004). The fragments size distribution function comes from a non-extensive Tsallis formulation, starting from first principles, i.e., a nonextensive formulation of the maximum entropy principle. This non-extensive approach leads to a G-R type law for the magnitude distribution of EQs:

$$
\begin{aligned}
& \log (N(M>))=\log N+\left(\frac{2-q}{1-q}\right) \times \\
& \log \left[1+\alpha(q-1) \times(2-q)^{(1-q) /(q-2)} \times 10^{2 m}\right]
\end{aligned}
$$

where $N$ is the total number of EQs, $N(M>)$ the number of EQs with magnitude larger than $M$, and $m \approx \log (\varepsilon)$.

This is not a trivial result, and incorporates the characteristics of nonextensivity into the distribution of EQs by magnitude. $\alpha$ is the constant of proportionality between the EQ energy, $\varepsilon$, and the size of fragment, $r$. More precisely, Sotongo and Posadas (2004) assume that $\varepsilon \propto r$.

Eq. (1) provides an excellent fit to seismicities associated with various seismic regions, while the corresponding $q$-values are distributed in the region $(1.60-1.70)$. Importantly, formula (1) also fits the sequence of precursory "electromagnetic earthquakes" under study very well, while the associated $q$ parameter is $q=1.80$ (Fig. 8). In Papadimitriou et al. (2008) a relevant detailed study has been presented. The similarity in the $q$-values is in harmony with the concept that the generation of a single EQ is a reduced image of the generation of the natural regional seismicity. Notice, the estimated values $q$-values (Kalimeri et al., 2008; Papadimitriou et al., 2008): (i) represent a subextensive system verifying the emergence of interactions and high organization during the activation of the fault; (ii) are in full agreement with the upper limit $q<2$ obtained from several independent studies involving the Tsallis nonextensive (Silva et al., 2006).

\section{Conclusions}

A vital problem in material science and in geophysics is the identification of precursors of macroscopic defects or shocks. In physics, the degree to which we can predict a phenomenon is often measured by how well we understand it. Despite the large amount of experimental data and the considerable effort that has been undertaken by the material scientists, many questions about the fracture remain standing. This fact is reflected in the dissapointing progress on short-term EQ prediction.

Fracture induced physical EM fields, rooted in the opening cracks, allow a real-time monitoring of damage evolution in materials during mechanical loading from the laboratory up to the geophysical scale. Clear kHz-to-MHz EM anomalies have been detected over periods ranging from a few days to a few hours prior to recent destructive EQs in Greece, with the $\mathrm{MHz}$ radiation appearing earlier than the $\mathrm{kHz}$. Recent results indicate that these EM time-series contain information characteristic of an ensuing seismic event (Kapiris et al., 2004a; Contoyiannis et al., 2005; Karamanos et al., 2006; Papadimitriou et al., 2008). An improved understanding of the EM precursors, especially its physical basis, has direct 
implications for the EQ generation processes and EQ prediction research. A challenge within this field is to distinguish characteristic epochs in the evolution of the precursory EM activity associated with the Athens EQ and identify them with the equivalent last stages in the EQ preparation process. In this direction, our model of the focal area consists of (i) a backbone of strong and large entities distributed along the activated fault and (ii) a strongly heterogeneous medium that surrounds the family of strong entities that prevent the free slip. Based on this model, we recently proposed the following two stages model (Contoyiannis et al., 2005; Papadimitriou et al., 2008): The first epoch, which includes the initially emerged $\mathrm{MHz}$ EM activity, originates during cracking in the highly heterogeneous material that surrounds the backbone of large and strong entities. This emission could be described in analogy with a thermal continuous phase transition. The second epoch includes the $\mathrm{kHz}$ EM radiation that emerges in the tail of the precursory EM activity and ceases approximately nine hours before the Athens EQ. This activity indicates an underlying nonequilibrium process without any footprint of an equilibrium thermal phase transition (Contoyiannis et al., 2005). This radiation is also characterized by the appearance of high organization and persistency (Karamanos et al., 2006; Kalimeri et al., 2008; Papadimitriou et al., 2008). We have attributed this radiation to the fracture of the backbone that sustains the system (Contoyiannis et al., 2005; Karamanos et al., 2006; Papadimitriou et al., 2008). Obviously, the above mentioned proposal needs further documentation. Thus, much more work is needed to improve the observations and refine the models of EM precursor generation. Physically, the appearance of persistency and organization may indicate that the process acquires a self-regulating character and to a great degree the property of irreversibility, one of the interesting components of predictive capability. Importantly, laboratory experiments of fracture (Mavromatou et al., 2004) suggest that the final pre-catastrophic stage is characterized by a dynamical instability which inevitably leads to fragmentation through: (i) successive strong catastrophic EM events, (ii) stress drop and (iii) a high increase of the first time derivative of stress (see Fig. 9).

In this work we attempt to further develop the above mentioned two stage model of the detected EM precursors. In particular, we focus on the second epoch / stage associated with $\mathrm{kHz}$ EM precursory emissions. The obtained results suggest the following:

1. The asperities of the Athens EQ might be damaged during the slipping of two rough and rigid fractionalBrownian-motion type profiles one over the other. This finding is in harmony with ample experimental and theoretical evidence.

2. Experimental results in very different types of materials seem to support the idea of a universal roughness exponent which is independent of the material properties.
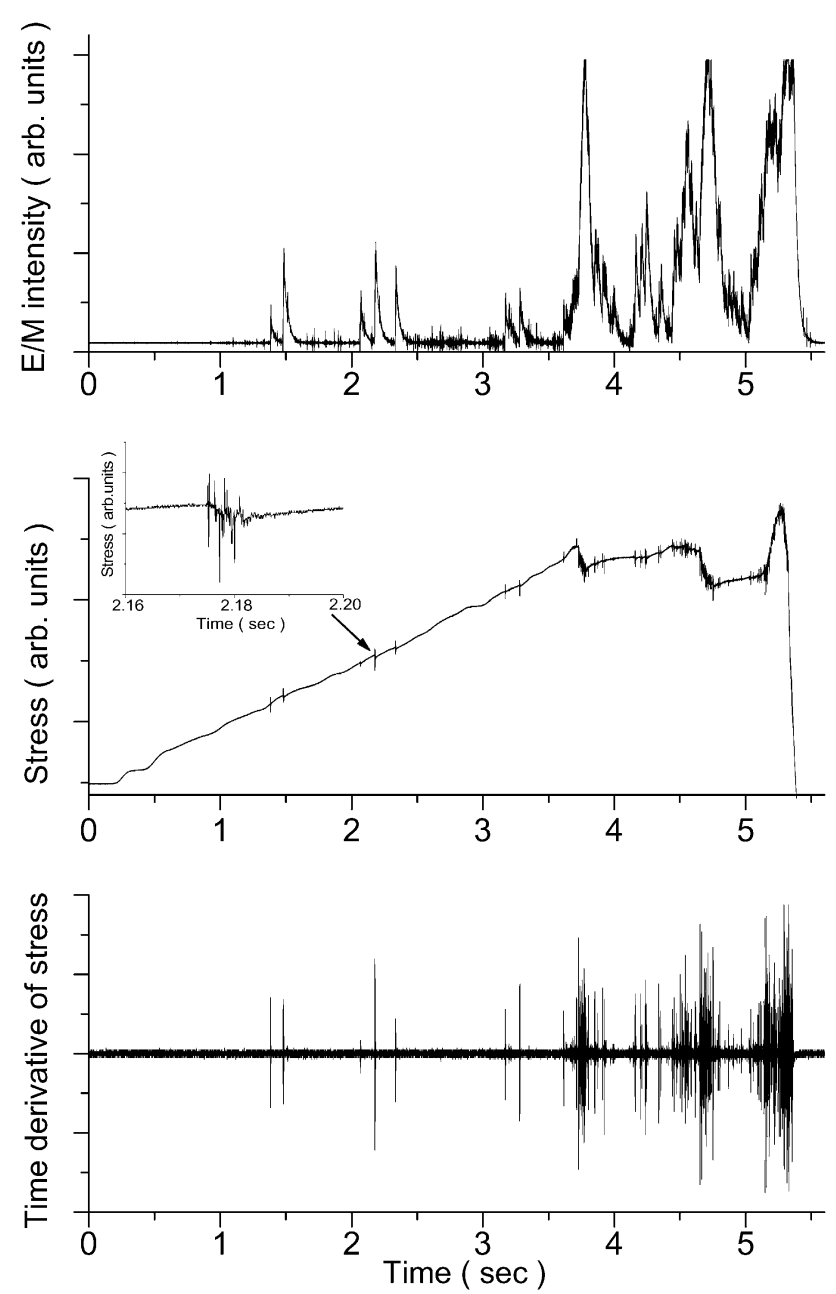

Fig. 9. The time evolution of EM field intensity (upper), stress (middle) and time derivative of stress (lower) up to the final failure of a granite sample (sampling rate: 20 ksamples/s (Mavromatou et al., 2004.

Indeed, the Hurst exponent $H \sim 0.75-0.8$ has been interpreted as a universal indicator of roughness of fracture surfaces, weakly dependent on the nature of the material. The roughness of the profile of the candidate EM precursor leads to a roughness of the fault surface which is compatible with the universal one.

3. The activation of a single EQ (fault) behaves as a reduced self-affine image of the whole regional seismicity and a magnified self-affine image of the laboratory seismicity. Importantly, Huang and Turcotte (1988) have pointed out that the statistics of seismicity could be merely a macroscopic reflection of the physical processes in EQ source. 

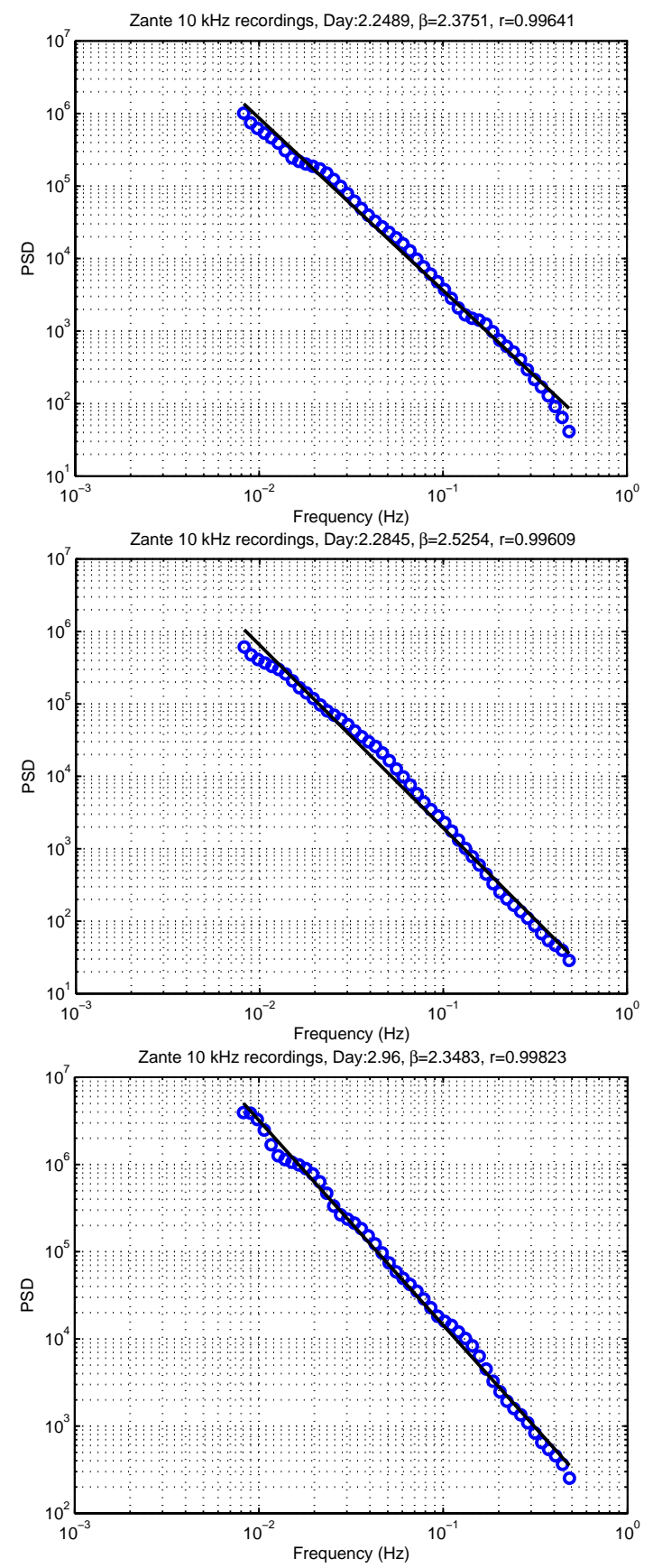

Fig. 10. The power spectrum density versus frequency in a $\log S(f)-\log f$ representation of three segments of the $10 \mathrm{kHz}$ EM emission recorded before the EQ that occurred on 14 February 2008 in southern Greece with magnitude 6.9. Each segment has a duration of $1024 \mathrm{~s}$ including 1024 points. The associated $\beta$-exponents leads to a roughness of the profile of the corresponding time series which is consistent with one of the profile of the $\mathrm{kHz}$ EM precursor associated with the Athens EQ (see Figs. 5, 6).
4. Miltenberger et al. (1993) and Carlson et al. (1994) have early pointed out that an important open question is whether the spatial and temporal complexity of EQs and fault structures emerge from geometrical and material built-in heterogeneities (Turcotte, 1997) or from the chaotic behavior inherent to the nonlinear equations governing the dynamics of these phenomena. The obtained results support the hypothesis that the complexity of fault nucleation emerges from geometrical heterogeneities.

New theoretical and experimental results support the above mentioned considerations. Irreversible thermodynamics theories with internal state variables can be used to derive a general constitutive law for both transient and steady-state behaviors of rocks (Kawada and Nagahama, 2006). Recently, irreversible thermodynamics applied to the damage mechanics reveals that the damage evolution produces the reported precursory temporal variations in EM radiation and mechanical energy releases prior to the Athens EQ (Kawada et al., 2007). Moreover, Muto et al. (2007), to investigate the relation between the rock friction and the fractal structure of preseismic electromagnetic radiations conducted a friction experiment simulating the motion of an asperity on a fault plane. From concepts on the fractal size-distribution and temporal evolution of fault asperities, the authors conclude that the frictional discharges occurring at asperities on the fault plane can be one of the origins of the pre-failure fractal EM radiations.

In this field of research we require the reproducibility of the results: the results based on $\mathrm{KHz}-\mathrm{MHz} E M$ data should be verified by independent experiments. This requirement is well-satisfied in the case of the Athens EQ. Indeed, the $\mathrm{kHz}$ EM activity associated with the Athens EQ is consistent with other precursors that are imposed by data from other disciplines such as: seismology in terms of cumulative Benioff "strain", infrared remote sensing, synthetic aperture radars interferometry, and ultra low-frequency seismic electric signals (SES) (Karamanos et al., 2006; Papadimitriou et al., 2008). Notice, these measurements could indicate the possible position of the epicenter and the magnitude of the impending EQ (Kapiris et al., 2005), while the analysis of $\mathrm{MHz}-\mathrm{KHz}$ EM emissions particularly point to way of estimating the time to global failure. As it is mentioned, many aspects of EQ phenomenology still escape our full understanding. The case of the Athens EQ suggests that: several kind of promising precursory changes do exist; through an intergrated search for various EQ precursors and of related physical mechanisms it is expected to lay foundation for EQ generation, and thus to achieve a better short-term EQ predictions.

It would be desirable to have the possibility to analyze more preseismic EM emissions. However, the collection of a volume of appropriate EM data for statistical purposes requires some decades of years at least. Due to their 
absorption, precursory $\mathrm{MHz}-\mathrm{kHz} \mathrm{EM}$ radiations are associated with surface EQs with magnitude 6 or larger which happen in the continental Greece or near the coastline. However, such as EQs occur in a very slow rate, so that a statistical evaluation stemming from the EM data is practically impossible. This fact obliges us to attempt a multidisciplinary evaluation of seismogenic origin of the detected candidate precursory EM emissions (Eftaxias et al., 2007b and references therein). Recently a strong EQ occurred on 14 February (10:09:22.5 UTC) in southern Greece $\left(36.57^{\circ} \mathrm{N}, 21.75^{\circ} \mathrm{E}\right)$ with a magnitude $M=6.9$. A sequence of strong $\mathrm{kHz} \mathrm{EM}$ avalanches has been detected on 8 and 9 February (Fig. 10). This EM activity has a long duration $(\sim 36 \mathrm{~h})$ and ceased $\sim 4$ days before the EQ. This activity shows the same behavior with the one associated with the Athens EQ, namely persistency and high organization. Characteristically, we observed: (i) A very important reduction of the Tsallis entropy inside the strong impulsive burst; the normalized q-Tsallis values are distributed around the value 0.65 as in the case of the Athens EQ (Kalimeri et al., 2008; Papadimitriou et al., 2008). Notice, the EM background is characterized by a normalized q-Tsallis entropy $\sim 0.95$, while the Tsallis entropy has been normalized with the q-Tsallis entropy for a uniform distribution of probabilities. (ii) The $H$-values into the emerged EM bursts are distributed around the value 2.5 (Fig. 10). We observe that the roughness of this new precursory EM time series is also in agreement with the universal value of the fracture surfaces. These new findings further support the results of the present study.

Acknowledgements. The project is co-funded by the European Social Fund and National Resources - (EPEAEK II) PYTHAGORAS (70/3/7357).

Edited by: M. Contadakis

Reviewed by: one anonymous referee

\section{References}

Akay, M.: Time Frequency and Wavelets in Biomedical Signal Processing Engineering, Wiley-IEEE Press, 768 pp., 1997.

Amaral, L., Goldberger, A., Ivanov, P., and Stanley, H.: ScaleIndependent Measures and Pathologic Cardiac Dynamics, Phys. Rev. Lett., 81, 2388-2391, 1998.

De Arcangelis, L., Hansen, A., and Herrmann, H.: Scaling laws in fracture, Phys. Rev. B, 40, 877-880, 1989.

Bahat, D., Rabinovitch, A., and Frid, V.: Tensile fracturing in rocks: Tectonofractographic and Electromagnetic Radiation Methods, Springer Verlag, Berlin, 570 pp., 2005.

Barriere, B. and Turcotte, D. L.: A Scale-Invariant CellularAutomata Model for Distribited Seismicity, Geophys. Res. Lett., 18, 2011-2014, 1991.

Benassi, A., Bertrand, P., Cohen, S., and Istas, J.: Identification of the Hurst index of a step fractional Brownian motion, Statistical Inference for Stochastic Processes, 3, 101-111, 2000.
Bhattacharyya, P.: Of overlapping Cantor sets and earthquakes: analysis of the discrete Chakrabarti-Stinchcombe model, Physica A, 348, 199-205, 2005.

Bouchon, M.: The state of stress on some faults of the San Andreas system as inferred from near-field strong motion data, J. Geophys. Res., 102, 11 731-11 744, 1997.

Carlson, M., Langer, J., and Shaw, B.: Dynamics of earthquake faults, Rev. Mod. Phys., 66, 657-670, 1994.

Chakrabarti, B. and Benguigui, L.: Statistical Physics of fracture and breakdown in disordered Systems, Oxford University Press, 176 pp., 1997.

Chakrabarti, B. and Stinchocombe, R.: Stick-slip statistics for two fractal surfaces: a model for earthquakes, Physica A, 270, 27-34, 1999.

Contoyiannis, Y., Kapiris, P., and Eftaxias, K.: Monitoring of a preseismic phase from its electromagnetic Precursors, Phys. Rev. E, 71, 066123, 1-14, 2005.

Dodze, D., Beroza, G., and Ellsworth, W.: Detailed observations of California foreshock sequences: implications for the earthquake initiation process, J. Geophys. Res., 101, 22 371-22 392, 1996.

Eftaxias, K., Kapiris, P., Polygiannakis, J., Bogris, N., Kopanas, J., Antonopoulos, G., Peratzakis, A., and Hadjicontis, V.: Signatures of pending earthquake from electromagnetic anomalies, Geophys. Res. Lett., 28, 3321-3324, 2001.

Eftaxias, K., Kapiris, P., Dologlou, E., Kopanas, J., Bogris, N., Antonopoulos, G., Peratzakis, A., and Hadjicontis, V.: EM anomalies before the Kozani-Grevena earthquake: A study of their behavior through laboratory experiments, Geophys. Res. Lett., 29, 691-694, 2002.

Eftaxias, K., Sgrigna, V., and Chelidze, T.: Mechanical and Electromagnetic Phenomena Accompanying Preseismic Deformation: from Laboratory to Geophysical Scale, Tectonophysics, 431, 1301, 2007a.

Eftaxias, K., Panin, V., and Deryugin Y.: Evolution EM-signals before earthquake and during laboratory test of rocks, Tectonophysics, 431, 273-300, 2007b.

Feng, X-T., and Seto, M.: Fractal structure of the time distribution of microfracturing in rocks, Geophys. J. Int., 136, 275-285, 1999.

Gusev, A.: On relation between earthquake population and asperity population on the fault, Tectonophysics, 211, 85-98, 1992.

Gutenberg, B. and Richter, C.: Seismicity of the Earth and Associated Phenomena, 2nd ed., 310 pp., Princeton Univ. Press, Princeton, New Jersey, USA, 1954.

Hainzl, S., Zoller, G., and Scherbaum, F.: Earthquake clusters resulting from delayed rupture propagation in finite fault segments, Geophys. Res. Lett., 108, 2013-2016, 2003.

Hallgass, R., Loreto, V., Mazzella, O., Paladin, G., and Pietronero, L.: Self-affine model of earthquakes, Phys. Rev. Lett., 76, 25992562, 1997.

Hansen, A., and Schmittbuhl, J.: Origin of the universal roughness exponent of brittle fracture surfaces: stress-weighted percolation in the damage zone, Phys. Rev. Lett., 90, 45 504-45 507, 2003.

Heneghan, C. and McDarby, G.: Establishing the relation between detrended fluctuation analysis and power spectral density analysis for stochastic processes, Phys. Rev. E, 62, 6103-6110, 2000.

Huang, J. and Turcotte, D.: Fractal distributions of stress and strength and variations of bvalue, Earth Planet. Sci. Lett., 91, 223-230, 1988. 
Kagan, Y. and Knopoff, L.: Spatial distribution of earthquakes: the two-point correlation function, Geophys. J. R. Astr. Soc., 62, 303-320, 1980

Kalimeri, M., Papadimitriou, C., and Eftaxias, K.: Dynamical complexity detection in pre-seismic emissions using nonadditive Tsallis entropy, Physica A, 387, 1161-1172, 2008.

Kapiris, P., Eftaxias, K., and Chelidze, T.: The electromagnetic signature of prefracture criticality in heterogeneous media, Phys. Rev. Lett., 92, 065702/1-4, 2004a.

Kapiris, P., Balasis, G., Kopanas, J., Antonopoulos, G., Peratzakis, A., and Eftaxias, K.: Scaling Similarities of Multiple Fracturing of Solid Materials, Nonlinear Proc. Geoph., 11, 137-151, 2004b.

Kapiris, P., Nomicos, K., Antonopoulos G., Polygiannakis J., Karamanos K., Kopanas J., Zissos A., Peratzakis A., and Eftaxias, K.: Distinguished seismological and electromagnetic features of the impending global failure: Did the 7/9/1999 M5.9 Athens earthquake come with a warning? Earth, Planets and Space, 57, 215230, 2005.

Karamanos, K., Peratzakis, A., Kapiris, P., Nikolopoulos, S., Kopanas, J., and Eftaxias, K.: Extracting Preseismic Electromagnetic Signatures in Terms of Symbolic Dynamics, Nonlinear Proc. Geoph., 12, 835-848, 2005.

Karamanos, K., Dakopoulos, D., Aloupis, K., Peratzakis, A., Athanasopoulou, L., Nikolopoulos, S., Kapiris, P., and Eftaxias, K.: Study of pre-seismic electromagnetic signals in terms of complexity, Phys. Rev. E, 74, 16104/1-21, 2006.

Kawada, Y. and Nagahama, H: Cumulative Benioff strain-release, modified Omoris law and transient behavior of rocks, Tectonophysics, 424, 157-166, 2006.

Kawada, Y., Nagahama, H., and Nakamura, N.: Time-scale invariant invariances in preseismic electromagnetic radiation, magnetization and damage evolution of rocks, Nat. Hazards Earth Syst. Sci., 7, 599-606, 2007,

http://www.nat-hazards-earth-syst-sci.net/7/599/2007/.

Kontoes, C., Elias, P., Sycioti, O., Briole, P., Remy, D., Sachpazi, M., Veis, G., and Kotsis, I.: Displacement field and fault model for the September 7, Athens earthquake inferred from the ers2 satellite radar interferometry, Geophys. Res. Lett., 27, 39893992, 2000.

Lei, X., Nishizawa, O., Kusunose, K., Cho, A., Satoh, T., and Nishizawa, O.: Compressive failure of mudstone samples containing quartz veins using rapid a monitoring: the role of asperities, Tectonophysics, 328, 329-340, 2000.

Lei, X., and Satoh, T.: Indicators of critical point behavior prior to rock failure inferred from pre-failure damage, Tectonophysics, 431, 97-111, 2007.

Li, H., Jia, Z., Bai, Y., Xia, M., and Ke, F.: Damage localization, sensitivity of energy release and the catastrophe transition, Pure Appl. Geophys., 159, 19331950, 2002.

Lopez, J. and Schmittbuhl, J.: Anomalous scaling of fracture surfaces, Phys. Rev. E, 57, 6405-6408, 1998.

Mandelbrot, B.: The Fractal Geometry of Nature, W. H. Freema, New York, USA, 486 pp., 1982.

Maslov, S., Paczuski, M., and Bak, P.: Avalanches and 1/f noise in evolution and growth models, Phys. Rev. Lett., 73, 2162-2165, 1994.

Mavromatou, C., Hadjicontis, V., Ninos, D., Mastrogiannis, D., Hadjicontis, E., and Eftaxias, K.: Understanding the fracture phenomena in inhomogeneous rock samples and ionic crystals, by monitoring the electromagnetic emission during their deformation, Phys. Chem. Earth, 29, 353-357, 2004.

Miltenberger, P., Sornette, D., and Vanneste, C.: Fault selforganization as optimal random paths selected by critical spatioremporal dynamics of earthquakes, Phys. Rev. Lett., 71, 36043607, 1993.

Mourot, G., Morel, S., Bouchaud, E., and Valentin, G.: Scaling properties of mortar fracture surfaces, Int. J. Fracture, 140, 3954, 2006.

Muto, J., Nagahama, H., Miura, T., and Arakawa, I.: Frictional discharge at fault asperities: origin of fractal seismoelectromagnetic radiation, Tectonophysics, 431, 113-122, 2007.

Papadimitriou, C., Kalimeri, M. and Eftaxias, K.: Nonextensivity and universality in the earthquake preparation process, Phys. Rev. E., 77, 036101/1-14, 2008.

Peyrat, S., Olsen, K., Madariaga, R.: Dynamic modeling of the 1992 Landers earthquake, J. Geophys. Res., 106, 26 467-26 482, 2001.

Ponomarev, A. Zavyalov, A., Smirnov, V., and Lockner, D.: Physical modelling of the formation and evolution of seismically active fault zones, Tectonophysics, 277, 57-81, 1997.

Ponson, L., Bonamy, D., and Bouchaud, E.: Two-dimensional scaling properties of experimental fracture surfaces, Phys. Rev. Lett., 96(3), 035506, doi:10.1103/PhysRevLett.96.035506, 2006.

Rabinovitch, A., Frid, V., and Bahat, D.: Gutenberg-Richter-type relation for laboratory fracture-induced electromagnetic radiation, Phys. Rev. E, 65, 11 401/1-11 401/4, 2001.

Rabinovitch, A., Frid, V., and Bahat, D.: Surface Oscillations A Possible Source of Fracture Induced Electromagnetic Radiation, Tectonophysics, 431, 15-22, 2007.

Reasenberg, P.: Foreshock occurrence rates before large earthquakes worldwide, Pure Appl. Geophys., 155, 355-379, 1999.

Renard, F., Voisin, C., Marsan, D., and Schmittbuhl, J.: High resolution 3D laser scanner measurements of a strike-slip fault quantity its morphological anisotropy at all scales, Geophys. Res. Lett., 33, L04305, 2006.

De Rubeis, V., Hallgass, R., Loreto, V., Paladin, G., Pietronero, L., and Tosi, P.: Self-affine model of earthquakes, Phys. Rev. Lett., 76, 2599-2562, 1996.

Rundle, J., Turcotte, D., Shcherbakov, R., Klein, W., and Sammis, C.: Statistical Physics approach to understanding the multiscale dynamics of earthquake fault systems, Rev. Geophys., 41(4), 1019, doi:10.1029/2003RG000135, 2003.

Sahimi, M., Robertson, M., and Sammis, C.: Fractal distribution of earthquakes hypocenters and its relation to fault patterns and percolation, Phys. Rev. Lett., 70, 2186-2189, 1993.

Sammonds, P. R., Meredith, P. G., and Main, I. G.: Role of pore fluid in the generation of seismic precursors to shear fracture, Nature, 359, 228-230, 1992.

Scholz, C.: The frequency-magnitude relation of macrofracturing in rocks and its relation to earthquakes, Bull. Seismo. Soc. Am., 58, 399-415, 1968.

Silva, R., Franca, G., Vilar, C., and Alcaniz, J.: Nonextensive models for earthquakes, Phys. Rev. E, 73, 026102/1-5, 2006.

Schorlemmer, D. and Wiemer, S.: Microseismicity data forecast rupture area, Nature, 434, doi:10.1038/4341086a, 2005. 
Solotongo-Costa, O. and Posadas, A.: Fragment-asperity interaction model for earthquakes, Phys. Rev. Lett., 92, 048501/1-4, 2004.

Sornette, D.: Critical Phenomena in Natural Sciences, Chaos, Fractals, Self-organization and Disorder: Concepts and Tools, Second edition, Springer Series in Synergetics, Heidelberg, 2004.

Sornette, D. and Andersen, J.-V.: Scaling with respect to disorder in tome-to-failure, Eur. Phys. J. B, 1, 353-357, 1998.

Turcotte., D.: Fractals and chaos in geology and geophysics, 2nd ed., 398 pp., Cambridge University Press, 1997.

Yulmetyev, R., Gafarov, F., Hanggi, P., Nigmatullin, R., and Kayunov, S.: Possibility between earthquake and explosion seismogram differentiation by discrete stochastic non-Markov processes and local Hurst exponent analysis, Phys. Rev. E, 64(6), 066132-1-066132-14, doi:10.1103/PhysRevE.64.066132, 2001.

Wiemer, S. and Schorlemmer, D.: ALM: An asperity-based likelihood model for California, Seismol. Res. Lett., 78, 134-140, 2007.

Zapperi, S., Kumar, P., Nukala, V., and Simunovic, S.: Crack roughness and avalanche precursors in the random fuse model, Phys. Rev. E, 71, 26106/1-10, 2005.

Zheng, G.-P. and Mo, L.: Dynamic scaling for avalanches in disordered systems, Phys. Rev. E, 63, 36122/ 1-6, 2001. 